\title{
An Integrated Workflow to Predict Macro-scale Transport Properties in Gas Shales by Coupling Molecular Dynamics Simulation with Lattice Boltzmann Method
}

\author{
Yang Ning ${ }^{1}$, Shuai $\mathrm{He}^{2}$ and Guan Qin ${ }^{3}$ \\ 1. University of Houston, Department of Chemical \& Biomolecular Engineering, Houston, USA. \\ 2. University of Houston, Department of Chemical \& Biomolecular Engineering, Houston, USA. \\ 3. University of Houston, Department of Petroleum Engineering, Houston, USA.
}

It is well known that shale formations exhibit multi-scale geological features such as nano-pores in formation matrix and natural fractures at multiple length scales [1]. The key challenge in unconventional reservoir simulations thus is how to preserve fine-scale information in coarse-scale reservoir simulations for correct production forecasting and reserve estimation [2]. In this paper, we present an upscaling workflow by coupling Molecular Dynamics (MD) simulation and lattice Boltzmann method (LBM) with digital rock reconstruction techniques to predict transport properties of natural gas in shales at different length scales. The proposed approach allows calculating macro-scale transport properties of natural gas in shales for unconventional reservoir simulations without losing critical fine-scale (nano-scale) information.

The reconstructions of multi-scale shale samples are performed using digital rock imaging techniques, i.e. FIB-SEM, Nano-CT, and Micro-CT. These experiments provide micro-scale pore architectures $(\sim \mathrm{nm})$, meso-scale mineralogical distribution $(\sim \mu \mathrm{m})$, and macro-scale natural-fracture network $(\sim \mathrm{cm})$, respectively. These multi-scale observations are then utilized for multi-scale digital rock reconstructions for the investigations of multi-scale transport properties. The present upscaling process can be summarized as three steps. First, micro-scale transport properties in organic and inorganic digital rocks from FIB-SEM are calculated using the non-equilibrium MD simulations. Representative organic (kerogen) and inorganic (montmorillonite, kaolinite, etc.) molecules are built upon their molecular structures. Transport properties determined from MD simulation are thenserved as input parameters for LBM simulations. Second, micro-scale properties of each component are mapped stochastically on its corresponding voxels in Nano-CT digital rocks. The meso-scale permeabilities of Nano-CT digital rocks are then calculated using the generalized LBM model. Last, the effective permeabilities of the macroscale shale samples (Micro-CT) with micro-fracture networks are calculated using the generalized LBM model, in which the matrix permeabilities are obtained from the step 2 . The analytical solutions of slip flows are utilized for flow in micro-fractures.

In the simulation results, the effective permeability changes significantly (order of magnitude) for shale samples at different scales. The difference in permeability is attributable mainly to porosity, organic content, and fracture characteristics. It is found that the effective permeability increases when the organic content is increased, and better pore connectivity in organic matters also enhances the effective permeability. In addition, the amount, aperture, and length of micro-fractures are found to have significant influences on the effective permeability.

Challenges associated with this upscaling workflow include: (1) micro-pores in kerogen and accurate nano-pore connectivity are difficult to obtain from the FIB-SEM due to the limits of imaging resolution, so we built a model for solid kerogen using the MD simulations that include micro-pores in order to enhance the pore connectivity. However, kerogen solids with the correct pore-size distribution are still 
challenging at this stage; (2) though organic matter and pyrite are easily distinguished, Nano-CT still has difficulties differentiating between inorganic minerals with similar density. This can be improved with the assistance of the XRD analysis; (3) Mapping from finer-scale to coarser-scale simulations is challenging. In this study, the simulation results in smaller-scale, as the mean values with a standard deviation, are mapped stochastically on the larger-scale simulation cells. The mapping can be improved by performing imaging experiments and flow simulations on more shale samples in smaller-scale to get more representative statistics.

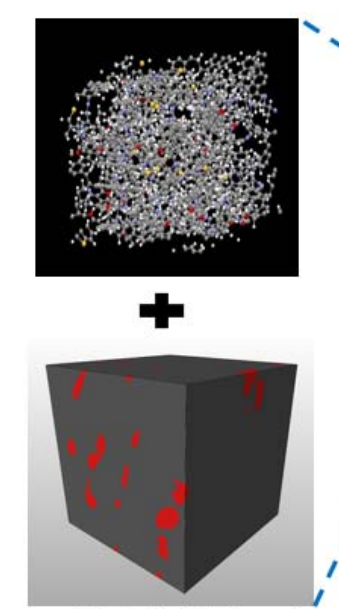

FIB-SEM (nm)

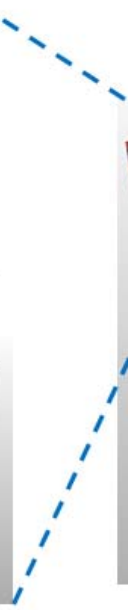$$
\text { (20) }
$$

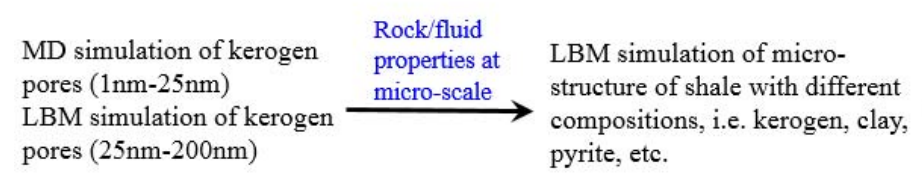

$$
\operatorname{NanoCT}(\mu \mathrm{m})
$$

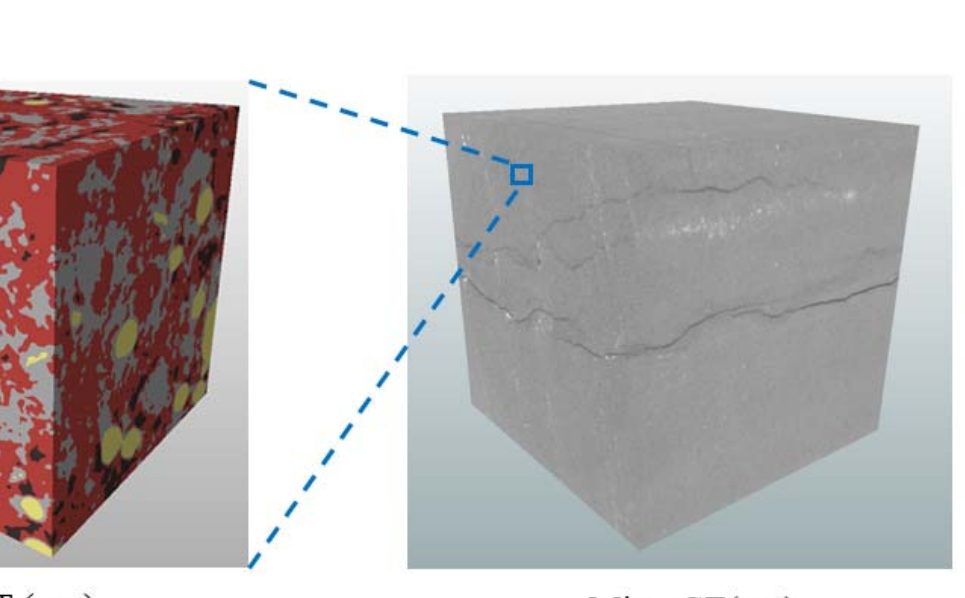

$\operatorname{MicroCT}(\mathrm{cm})$

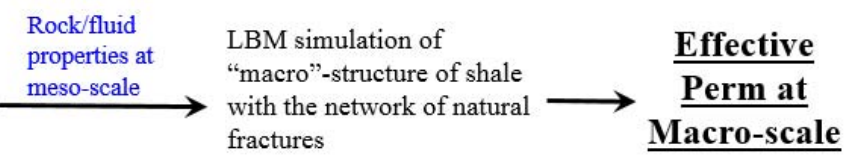

Figure 1. Schematic illustration of the integrated upscaling workflow. Left top: Kerogen molecular structure $(3.5 \mathrm{~nm} \times 3.5 \mathrm{~nm} \times 3 \mathrm{~nm})$ using MD simulations; Left bottom: FIB-SEM digital rock of kerogen $\left(1.5 \mu \mathrm{m}^{3}\right)$; Middle: Nano-CT digital rock of shale matrix $\left(41 \mu \mathrm{m}^{3}\right)$; Right: Micro-CT digital rock with natural fracture network $\left(1.5 \mathrm{~cm}^{3}\right)$.

\section{References:}

[1] Ayaz Mehmani, Maša Prodanović and Farzam Javadpour (2013), Multiscale, Multiphysics Network Modeling of Shale Matrix Gas Flows, Transport in Porous Media, 99, 2, page number 377-390. [2] Yang Ning, Shuai He, Honglin Liu, Hongyan Wang and Guan Qin, Upscaling in Numerical Simulation of Shale Transport Properties by Coupling Molecular Dynamics Simulation with Lattice Boltzmann Method, URTeC 2459219, San Antonio, Texas, USA, August 1-3, 2016 